These differed between ACPA-subgroups, implying a different interaction of synovium and bone in these patients.

Disclosure of Interests: None declared

DOI: 10.1136/annrheumdis-2020-eular.2157

\section{OP0177 PROPERTIES AND LOCATIONS OF COLOR-CODED DUAL ENERGY CT LESIONS IN GOUT PATIENTS - A SYSTEMATIC EVALUATION}

S. Nysom Christiansen ${ }^{1}$, F. C. Müller ${ }^{2}$, M. Ǿstergaard ${ }^{1}$, O. Slot ${ }^{1}$, J. Møllenbach Møller ${ }^{2}$, H. F. Børgesen ${ }^{2}$, K. K. Gosvig ${ }^{2}$, L. Terslev ${ }^{1} .{ }^{1}$ Rigshospitalet,

Copenhagen Center for Arthritis Research, Center for Rheumatology and Spine Diseases, Glostrup, Denmark; ${ }^{2} \mathrm{Herlev}$ Hospital, Department of Radiology, Herlev, Denmark

Background: Dual energy CT (DECT) has diagnostic potential in gout patients. DECT can automatically colour-code presumed urate deposits based on radiodensity (Hounsfield Units, HU) and DECT ratio (difference in attenuation between high and low $\mathrm{kV}$ series) of lesions. However, other materials may imitate properties of urate deposits, most importantly calcium-containing material, dense tendons and artefacts, which may lead to misinterpretations. The characteristics of DECT lesions in gout patients have not yet been systematically investigated. Objectives: To evaluate the properties and locations of colour-coded DECT lesions in gout patients.

Methods: DECT were performed in patients with suspected gout. Patients were separated into gout and non-gout patients based on joint fluid microscopy findings. DECT of the hands, knees and feet were performed using default gout settings and colour-coded lesions were registered. Only location-relevant lesions were analysed (e.g. nail bed artefacts excluded). Mean density (mean of $\mathrm{HU}$ at $80 \mathrm{kV}$ and Sn150kV), mean DECT ratio, size and location of each lesion was determined. Subgroup analysis was performed post-hoc evaluating potential differences in properties and locations of lesions. Lesions were separated into groups according to properties (Figure 1, grey box): 1) Size - to separate artefacts characterised by small volume (possible artefacts). 2) DECT ratios-to separate calcium-containing material characterised by high DECT ratio (possible calcium-containing material). 3) Density - to separate dense tendons characterised by low DECT ratio and low $\mathrm{HU}$ values (possible dense tendons). Lesion fulfilling all urate characteristics (large volume, low DECT ratio, high density) were labelled definite urate deposits. Finally, for non-gout patients, properties of non-gout urate-imitation lesions (properties as definite urate deposits) were analysed.

Results: In total, 3918 lesions (all lesions) were registered in gout patients $(n=23)$, with mean DECT ratio 1.06 (SD 0.13 ), median density $160.6 \mathrm{HU}$ and median size 6 voxels (Figure 1, blue box). Lesions were seen in all analysed joints, most frequently MTP1 joints (medial side), knee joints and midtarsal joints (Figure 2a). Tendon affections were also common, especially in the knee tendons (patella and quadriceps), malleolus-related tendons (e.g. peroneus and tibialis posterior) and the Achilles tendons (Figure 2a).

Subgroup analyses showed that definite urate deposits (figure $2 \mathrm{~b}$ ) were found at the same locations as all lesion in gout patients (figure 2a), with the four most common sites being MTP1 joints, midtarsal joints, and quadriceps and patella tendons (Figure 2b). Possible dense tendon lesions had a mean $\mathrm{HU}$ value of 156.5 $\mathrm{HU}-$ markedly higher than expected for dense tendons $(<100 \mathrm{HU})$-and lesion-locations were similar to definite urate deposits (data not shown), indicating that they primarily consisted of true urate deposits. In contrast, possible calcium-containing material and non-gout urate-imitating lesions had distinctly different properties (ratios 1.33 and 1.20, respectively) (Figure 1, yellow and orange box). Furthermore, the locations of these lesions were different from definite urate deposits since they were primarily found in a few weight-bearing joints (knee, midtarsal and talocrural including malleolus regions) and tendons (Achilles and quadriceps), whereas no lesions were found in either MTP1 joints or patella tendons (figure 2c).

Conclusion: DECT color-coded lesions in gout patients are heterogeneous in properties and locations. Subgroup analyses found that locations such as MTP1 joints and patella tendons were characterised by almost only showing definite urate deposits. A sole focus on these regions in the evaluation of gout patients may therefore improve specificity of DECT scans.

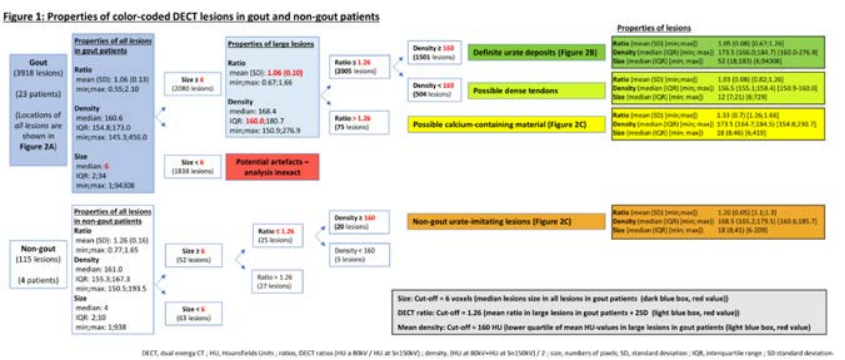

Figure 2

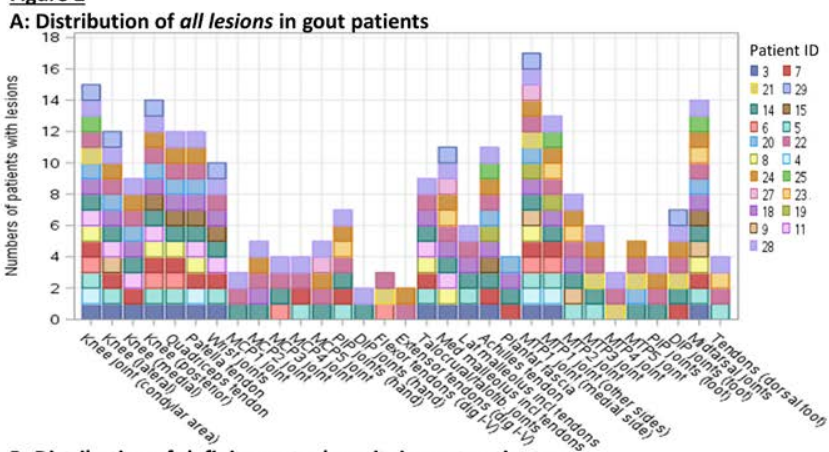

B: Distribution of definite urate deposits in gout patients

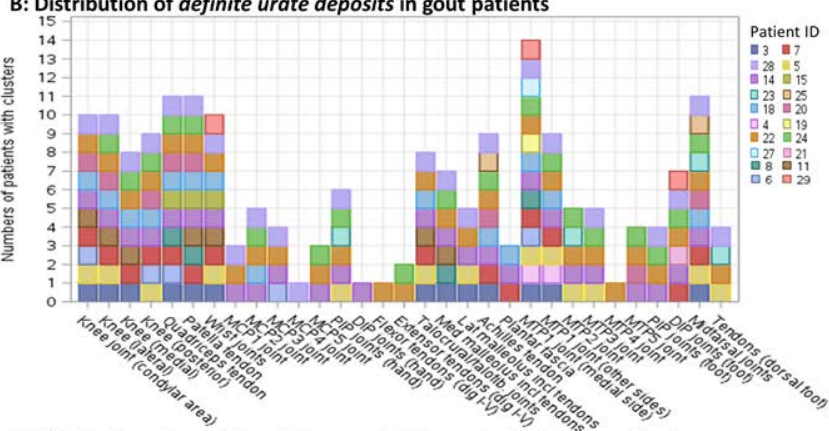

C: Distribution of possible calcium-containing material in gout patients and non-gout urate-imitating lesions

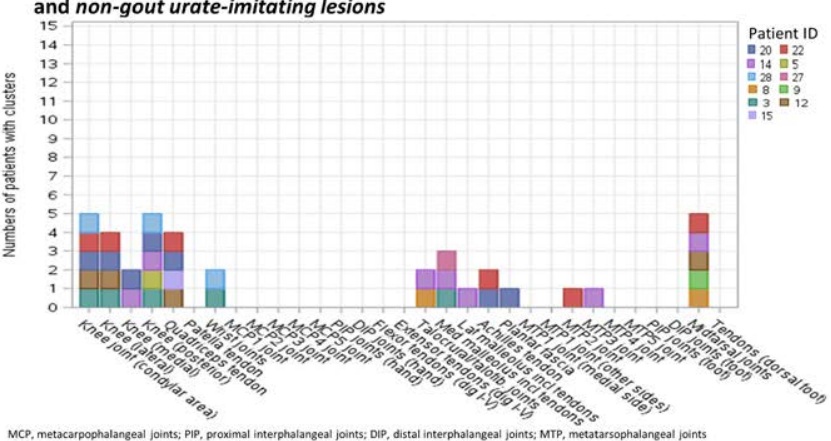

Disclosure of Interests: Sara Nysom Christiansen Speakers bureau: SNC has received speaker fees from Bristol Myers Squibb (BMS) and General Electric (GE)., Felix C Müller Employee of: Siemens Healthineers., Mikkel Ǿstergaard Grant/research support from: AbbVie, Bristol-Myers Squibb, Celgene, Merck, and Novartis, Consultant of: AbbVie, Bristol-Myers Squibb, Boehringer Ingelheim, Celgene, Eli Lilly, Hospira, Janssen, Merck, Novartis, Novo Nordisk, Orion, Pfizer, Regeneron, Roche, Sandoz, Sanofi, and UCB, Speakers bureau: AbbVie, Bristol-Myers Squibb, Boehringer Ingelheim, Celgene, Eli Lilly, Hospira, Janssen, Merck, Novartis, Novo Nordisk, Orion, Pfizer, Regeneron, Roche, Sandoz, Sanofi, and UCB, Ole Slot: None declared, Jakob Møllenbach Møller: None declared, Henrik F Børgesen: None declared, Kasper K Gosvig: None declared, Lene Terslev Speakers bureau: LT declares speakers fees from Roche, MSD BMS, Pfizer, AbbVie, Novartis, and Janssen. DOI: 10.1136/annrheumdis-2020-eular.1757

\begin{tabular}{l|l}
\hline OP0178 & IMAGING NEOANGIOGENESIS IN RHEUMATOID \\
ARTHRITIS (INIRA): WHOLE-BODY SYNOVIAL UPTAKE \\
OF A 99MTC-LABELLED RGD PEPTIDE IS HIGHLY \\
CORRELATED WITH POWER DOPPLER ULTRASOUND
\end{tabular}

L. Attipoe ${ }^{1}$, S. Subesinghe ${ }^{2}$, C. Blanco-Gil ${ }^{2}$, M. Opena ${ }^{2}$, M. Rosser ${ }^{3}$, G. Cook $^{1}$, A. Cope ${ }^{1}$, T. Garrood ${ }^{2}{ }^{1}$ King's College London, London, United Kingdom; ${ }^{2}$ Guy's and St Thomas' NHS Foundation Trust, London, United Kingdom; ${ }^{3}$ Serac Life Sciences, London, United Kingdom

Background: Power Doppler ultrasound (PDUS) is superior to clinical examination in detecting synovitis in patients with rheumatoid arthritis (RA). Although dynamic and cheap it is impractical to scan large numbers of joints in routine clinical settings. MRI, whilst sensitive for synovitis, is expensive and routine use is limited to targeted joints. Bone scintigraphy produces whole body images but due to limited specificity is not routinely used. 
${ }^{99 m}$ Tc-maraciclatide (Serac Healthcare) is a radiolabelled tracer which binds with high affinity to integrin $\alpha_{v} \beta_{3}$, a cell-adhesion molecule up-regulated on neoangiogenic blood vessels. It therefore has the potential to image synovial inflammation at the whole-body level. We previously showed in a pilot study that uptake was seen in the inflamed joints of five RA patients and that this correlated with PDUS. This study explores correlation with PDUS in a larger groups of patients with varied disease activity.

Objectives: To determine the correlation between ultrasound and ${ }^{99 \mathrm{~m}} \mathrm{Tc}-\mathrm{marac}-$ iclatide imaging in patients with rheumatoid arthritis.

Methods: 50 patients with RA fulfilling ACR 2010 classification criteria were recruited. Patients underwent an ultrasound scan of 40 joints with grey scale (GS) and PD quantification. Each joint was scored on a scale of 0-3 for GS and PD with a total score calculated for each patient. Within 3 hours of the ultrasound patients were injected with $740 \mathrm{MBq}$ of ${ }^{99 \mathrm{~m}} \mathrm{Tc}$-maraciclatide. Using a gamma camera, whole body planar views and dedicated hand and foot views were taken 2 hours after injection (Figure 1). Acquisition time was 20 minutes for whole body and 20 minutes for hand and foot views.

${ }^{99 \mathrm{~m}}$ Tc-maraciclatide images were scored as positive or negative uptake for each joint (binary score). A quantitative score was also calculated for each joint where there was uptake with this corrected for background uptake. Total binary and quantitative scores per patient were calculated.

Ultrasound and ${ }^{99 \mathrm{~m}} \mathrm{Tc}$-maraciclatide scores were tested for correlation with Pearson's correlation coefficient ( $r$ ). Interrater agreement for 2 scorers was calculated using kappa $(\mathrm{k})$ and concordance correlation coefficient $(P C)$.

Results: Strong correlation was seen when total PDUS was compared to binary scores $\left(r=0.92, r^{2}=0.85\right)$ (Figure 2) and quantitative scores $\left(r=0.85, r^{2}=0.72\right) . k$ was 0.82 and 0.79 for binary and ultrasound scores respectively. $P C$ was 0.82 for quantitative scores. p was $<0.0005$ for all results. ${ }^{99 \mathrm{~m}} \mathrm{Tc}$-maraciclatide uptake was also seen in inflamed tendons/tendon sheaths. The imaging procedure was well-tolerated. There were no tracer-related adverse events.

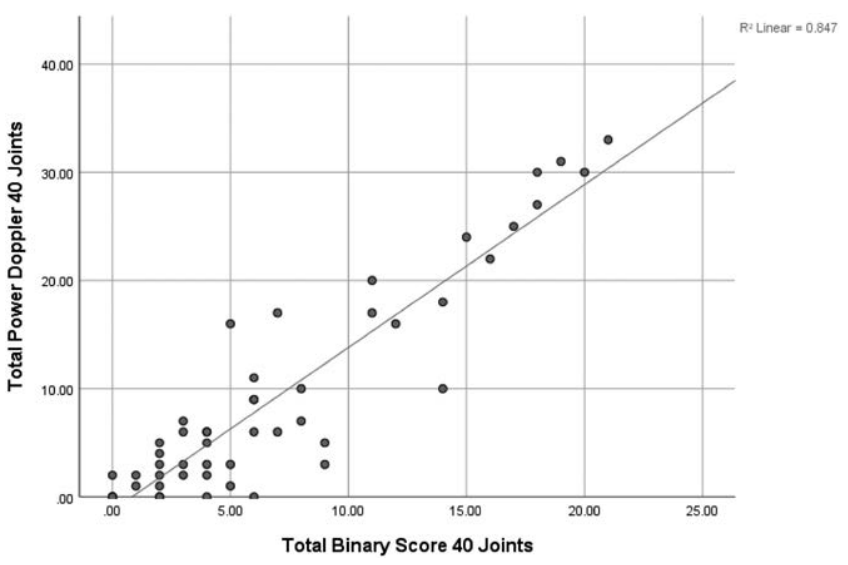

Figure 1. ${ }^{99 \mathrm{~m}} \mathrm{Tc}$-maraciclatide imaging with dedicated hand and foot views

Conclusion: ${ }^{99 \mathrm{~m}} \mathrm{Tc}$-maraciclatide uptake was highly correlated with PDUS highlighting its potential as an alternative imaging modality. ${ }^{99 \mathrm{~m}} \mathrm{Tc}$-based planar imaging has the unique capacity to image the whole body and hence the total synovial inflammatory load in a quick acquisition. The imaging equipment to perform these scans is already widely available in radiology departments. Interpretation of scans is also much simpler compared to US/MRI. It could therefore have a role in key decision-making points in pathways for diagnosis, treatment failure, and remission prior to dose tapering.

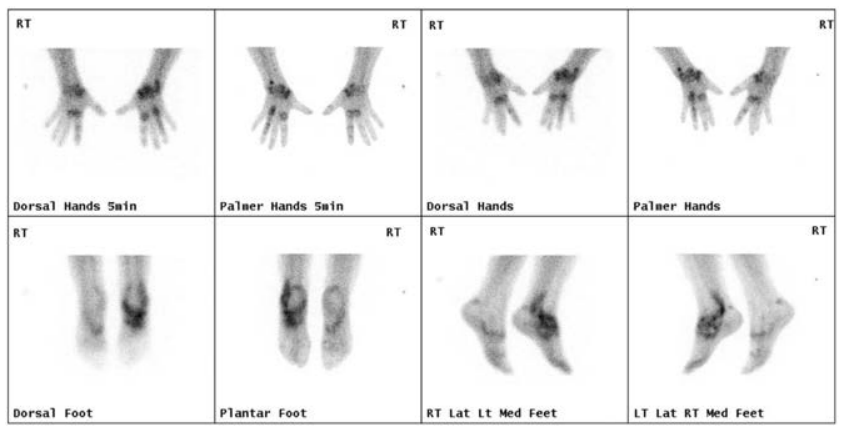

Figure 2. Correlation between total power doppler and ${ }^{99 \mathrm{~m}} \mathrm{Tc}$-maraciclatide binary scores
Disclosure of Interests: None declared

DOI: 10.1136/annrheumdis-2020-eular.5482

\section{OP0179 \\ USEFUL STUDY I: A MULTICENTRE LONGITUDINAL STUDY TO TEST WHETHER ULTRASOUND CAN IDENTIFY PATIENTS WITH MUSCULOSKELETAL SYMPTOMS OF LUPUS WITH BETTER RESPONSE TO THERAPY}

K. Mahmoud ${ }^{1}$, A. Zayat ${ }^{2}$, M. Y. MD Yusof ${ }^{1}$, K. Dutton ${ }^{1}$, L. S. Teh ${ }^{3}$, C. S. Yee ${ }^{4}$, D. D'cruz ${ }^{5}$, N. Ng ${ }^{5}$, D. Isenberg ${ }^{6}$, C. Ciurtin ${ }^{6}$, P. G. Conaghan ${ }^{1}$, P. Emery ${ }^{1}$, C. Edwards ${ }^{7}$, E. Hensor ${ }^{1}$, E. Vital ${ }^{1} .{ }^{1}$ University of Leeds and NIHR Leeds Biomedical Research Centre, Leeds, United Kingdom; ${ }^{2}$ Bradford Teaching Hospitals NHS Foundation Trust, Bradford, United Kingdom; ${ }^{3}$ Royal Blackburn Teaching Hospital and University of Central Lancashire, Blackburn, United Kingdom; ${ }^{4}$ Doncaster and Bassetlaw NHS Trust, Doncaster, United Kingdom; ${ }^{5}$ Guys and St Thomas Hospital, London, United Kingdom; ${ }^{6}$ University College London, London, United Kingdom; ${ }^{7}$ University Hospital Southampton NHS Foundation Trust, Southampton, United Kingdom

Background: In SLE, musculoskeletal manifestations impact on quality of life and trial outcomes. We previously showed that assessments based on joint swelling lack sensitivity, specificity and responsiveness compared to ultrasound (US).

Objectives: To determine clinical features predicting US synovitis and whether patients with US synovitis respond better to therapy

Methods: SLE patients were recruited if the referring physician deemed they had inflammatory pain warranting treatment. Swollen joints were not required. At baseline, physicians recorded features of inflammation, concurrent fibromyalgia and osteoarthritis. Stable doses of prednisolone ( $\leq 5 \mathrm{mg} /$ day), antimalarials or immunosuppressants were allowed. Participants received depomedrone 120mg IM then were assessed at 0,2 and 6 weeks for 66/68 swollen and tender joint counts, BILAG-2004, SLEDAI-2K, physician global and MSK-VAS, inflammatory markers, patient pain and disease activity-VAS, HAQ-DI, LupusQoL, US of hands and wrists (blinded to patient and clinical assessor). An internal pilot determined the primary endpoint:(Early Morning stiffness-VAS (EMS-VAS) at 2 weeks (adjusted for baseline) between patients with US-synovitis (GS $\geq 2$ or $P D \geq 1$ in $\geq 1$ joint) vs. normal US at baseline. $20 \%$ difference was considered clinically meaningful. Sensitivity analyses adjusted for prednisolone and immunosuppressants. Results: 122/133 patients completed all visits. There was significant disagreement between clinical examination and US. $78 / 133$ had US synovitis; $68 \%$ of these had $\geq 1$ swollen joint. Of $66 / 133$ patients with $\geq 1$ swollen joint, $20 \%$ had normal US. US-synovitis was more likely with joint swelling, a symmetrical small joint distribution and active serology. Physician-determined EMS, other lupus features or prior response to therapy were not associated. Fibromyalgia or osteoarthritis did not reduce the probability of US synovitis.

In the full analysis set $(n=133)$ there was no difference in EMS VAS at 2 weeks according to US synovial status at baseline (difference $-8 \mathrm{~mm}, 95 \% \mathrm{Cl}-19,4 \mathrm{~mm}$, $p=0.178)$. 32 patients had fibromyalgia. After excluding them, we found a statistically and clinically significantly better clinical response to depomedrone in patients with US-synovitis at baseline (baseline-adjusted EMS VAS at 2 weeks $-12 \mathrm{~mm}, 95 \% \mathrm{Cl}-24,0 \mathrm{~mm}, \mathrm{p}=0.049)$. This difference was greater in the treatment-adjusted sensitivity analysis $(-12.8(95 \% \mathrm{Cl}-22,-3 \mathrm{~mm}), \mathrm{p}=0.007)$ and the per-protocol-adjusted sensitivity analysis $(-14.8 \mathrm{~mm}(95 \% \mathrm{Cl}-20.8,-8.8 \mathrm{~mm})$ $\mathrm{p}<0.001)$. Patient with US synovitis had higher rates of improvement in the MSK BILAG-2004 (56\% vs. $26 \%, p=0.09$ ) and SLEDAI-2K (37\% vs. 15\%, $p=0.03$ ).

Conclusion: In lupus arthritis, distribution and serology, but not other features, help identify US-synovitis. US-synovitis was independent of features of fibromyalgia, but fibromyalgia confounded assessment of clinical response. US should be used to select SLE arthritis patients for therapy and clinical trials, especially when there are inflammatory symptoms without swollen joints.

Acknowledgments: The Project was funded by Lupus-UK

Disclosure of Interests: Khaled Mahmoud: None declared, Ahmed Zayat: None declared, Md Yuzaiful Md Yusof: None declared, Katherine Dutton: None declared, Lee-Suan Teh: None declared, Chee-Seng Yee: None declared, David d'cruz Grant/research support from: GlaxoSmithKline, Nora Ng: None declared, David Isenberg Consultant of: Study Investigator and Consultant to Genentech, Coziana Ciurtin Grant/research support from: Pfizer, Consultant of: Roche, Modern Biosciences, Philip G Conaghan Consultant of: AbbVie, BMS, Eli Lilly, EMD Serono, Flexion Therapeutics, Galapagos, GSK, Novartis, Pfizer, Speakers bureau: AbbVie, Eli Lilly, Novartis, Pfizer, Paul Emery Grant/research support from: AbbVie, Bristol-Myers Squibb, Merck Sharp \& Dohme, Pfizer, Roche (all paid to employer), Consultant of: AbbVie (consultant, clinical trials, advisor), Bristol-Myers Squibb (consultant, clinical trials, advisor), Lilly (clinical trials, advisor), Merck Sharp \& Dohme (consultant, clinical trials, advisor), Novartis (consultant, clinical trials, advisor), Pfizer (consultant, clinical trials, advisor), Roche (consultant, clinical trials, advisor), Samsung (clinical trials, advisor), Sandoz (clinical trials, advisor), 